\title{
A COMPARISON OF METHODS FOR PROPAGATING SURFACE DEFORMATION UNCERTAINTIES IN MODEL PARAMETERS
}

\author{
Wojciech GRUSZCZYŃSKI, Zygmunt NIEDOJADLO and Dawid MROCHEŃ * \\ AGH University of Science and Technology, Faculty of Mining Surveying and Environmental Engineering, \\ Al. Mickiewicza 30, 30-059 Cracow, Poland
}

*Corresponding author's e-mail: dawmro@agh.edu.pl

\begin{tabular}{l}
\hline ARTICLE INFO \\
\hline Article history: \\
Received 13 May 2019 \\
Accepted 23 August 2019 \\
Available online 17 October 2019
\end{tabular}

Keywords:

Monte Carlo simulation

Uncertainty propagation

Mining deformation

Indicators

\begin{abstract}
Two commonly utilized methods for propagating uncertainties in model parameters of surface deformation caused by underground mining are compared in this study. These methods enable estimation of standard uncertainties in indicators of surface deformation, specifically: subsidence, tilt, and horizontal strain, to be calculated. The first method is the law of propagation of uncertainty (LPU), based on a first-order Taylor series expansion without the use of higherorder derivatives. The second method is the Monte Carlo (MC) method. It relies on the calculation of many values of deformation indicator for random values of model parameters. It is noteworthy that in the previous research in this field no comparison of these methods was carried out. The calculations performed in this study based on both methods allow the verification of results and the features of both approaches to be compared. Calculations were performed applying the Knothe model, which assumes a normal distribution for the influence function. Both average values and model parameter uncertainties derived from hard coal mining across the entire Upper Silesian Coal Basin (USCB) region of Poland were used in this study. Model parameter uncertainties are high in this case, which is unfavorable for LPU, but results do indicate that this approach can be useful for propagating model parameter uncertainties for the surface deformation caused by underground mining.
\end{abstract}

\section{INTRODUCTION}

Deformation indicator value predictions are usually made incorporating a certain margin of error that includes consideration of the uncertainty with which they are determined. This margin of error should be as low as possible for economic reasons, while at the same time must be large enough to ensure the safety of surface structures adjacent to mining activity.

Sources of prediction uncertainty include:

- Model deficiencies. In general, predictive models assume that the properties of rock masses are simple and (frequently) uniform, and do not commonly take into account parameter variations or tectonics, which can lead to systematic discrepancies between calculated and observed indicator values;

- Random scatter in deformation indicators. The inherent nature of predictive models means that they tend to generate average deformation indicators under specific conditions, and do not consider random scatter, and;

- Uncertainty in model parameter values. The model parameters used for calibration are generally determined based on available information, most often historical data for the exploration area.

The last of these three main sources of uncertainty is directly correlated both with forecasting and the scope of available information. The aim of this paper is therefore to compare and contrast two commonly applied methods that facilitate the propagation of parameter uncertainties when calculating values for deformation indicators. These methods are the law of propagation of uncertainty (LPU; Taylor, 1997) and the Monte Carlo simulation (MC; Metropolis and Ulam, 1949). Conducted analysis assumes that parameter uncertainties are known; thus, the quantification and propagation of model parameter uncertainties means that they can be included within deformation indicator predictions. As a result, it is possible to rationally determine the margin with which the forecast is made. Previous research in this field (Hejmanowski and Malinowska, 2016; Kowalski, 2005; Kwinta, 2012; Niedojadło, 2008; Niedojadło and Gruszczyński, 2015) has tended to use just one of the methods available and so no comparative studies have so far been presented. 
The presented research results are a continuation of the authors' work on determining the uncertainty of parameters of the surface deformation model (Gruszczyński et al., 2018; Gruszczyński et al., 2019). W. Gruszczyński's program was used to calculate the values of indicators and their statistics estimated on the basis of the Monte Carlo method and the law of propagation of uncertainty, while the ISAAC library written by W. Ehrhardt was used to generate pseudorandom numbers. The Statistica program (ver. 13) was used to analyze the convergence of calculations and to make charts of random variable distributions.

\section{MATERIALS AND METHODS}

\subsection{METHOD FOR PREDICTING DEFORMATION}

In the research all the deformation indicators calculations were conducted with use of Knothe model (Knothe, 1957). This model belongs to the influence function group and it is based on the assumption of a normal distribution of influence function, given by the Equation 1:

$G(x, \mathrm{y})=\frac{a \cdot g}{r^{2}} \cdot \exp \left(-\pi \cdot \frac{x^{2}+y^{2}}{r^{2}}\right)$

where: $a$ denotes the exploitation coefficient, $g$ is operating height, $r$ is the radius of main influence, $x$ and $\mathrm{y}$ are the point coordinates at which the influence is calculated. The radius $r$ is calculated in accordance to Equation 2:

$$
r=\frac{H}{\tan \beta}
$$

where: $H$ is a depth of excavation, and $\tan \beta$ is a parameter describing dispersion of influences for a given rock mass.

Subsidence $s$ (3) and deformation indicators i.e. tilts $T$ (4) and horizontal strain $E$ (5) are therefore expressed as follows:

$$
\begin{aligned}
& s(x, \mathrm{y})=\frac{a \cdot g}{r^{2}} \iint_{p} \exp \left(-\pi \cdot \frac{x^{2}+y^{2}}{r^{2}}\right) d P \\
& T_{x}(x, \mathrm{y})=\frac{d s}{d x}, \quad T_{y}(x, \mathrm{y})=\frac{d s}{d y} \\
& E_{x}(x, \mathrm{y})=-B \frac{d^{2} s}{d x^{2}}, E_{y}(x, \mathrm{y})=-B \frac{d^{2} s}{d y^{2}}, \\
& E_{x y}(x, \mathrm{y})=-B \frac{d^{2} s}{d x d y}
\end{aligned}
$$

Parameter values can be determined by analyzing previous exploitations of a given mining area (Kowalski, 2005) or from on-going surveys along an observation line (Kwinta, 2012). The applied Knothe model therefore utilizes four parameters:
- exploitation coefficient $a$,

- main influence angle tangent $\tan \beta$,

- $\quad$ operating $\operatorname{rim} p$, and

- horizontal deformation coefficient $B$ given by Equation 6 , where $b_{0}$ is the proportionality factor

$B=b_{0} \cdot r$

The parameter values are assumed to be constant over time as exploitation progresses, although changes are allowed as a result of multiple mining excavations. This is also likely to be the case due to potentially residual influences derived from previous exploitation events, or changes in the geomechanical properties of rocks.

\subsection{APPLIED METHODS FOR PROPAGATING UNCERTAINTY}

Although a range of methods are available for propagating uncertainties in model parameters (Aien et al., 2016; Aien et al., 2014; Hejmanowski and Malinowska, 2016; Kowalski, 2005; Naworyta et al., 2005; Niedojadło and Gruszczyński, 2015; Soroudi and Amraee, 2013), the LPU approach and MC simulations are compared in this study. These methods enable different possibilities and necessitate various computational loads. The advantages and disadvantages of using both methods are discussed in the previous articles (Gruszczyński et al., 2018; Gruszczyński et al., 2019).

The LPU and MC methods were applied in this study assuming knowledge about parameter uncertainties. The assumptions of existence of model parameter values that do not incorporate systematic discrepancies between predicted and observed deformation were made. All discrepancies between model and observations are therefore considered to be uncorrelated noise with an average of zero.

\subsection{EXPERIMENT SETUP}

All the calculations reported in this study were performed via simulated exploitation of the Knothe model. The simulated exploitation used in this study to generate surface deformations is shown in Figure 1.

The simulated exploitation employed in this study included three walls excavated with caving. In each case the longwall had a width of $250 \mathrm{~m}$ and a length of $900 \mathrm{~m}$. The excavation took place at a depth of $600 \mathrm{~m}$ and had height of $2.5 \mathrm{~m}$. For the sake of simplicity of analysis, the coal seam was simulated as horizontal.

Values for deformation indicators and their uncertainties were modeled for points located on the line that crosses from west-to-east via the center of the wall block (marked $\mathrm{W}-\mathrm{E}$ in Fig. 1). This line exceeds the outline of the longwall by $400 \mathrm{~m}$ and reaches the center of wall $\mathrm{B}$ to ensure that calculated deformation indicators encompass a whole range that result from this simulated exploitation. 


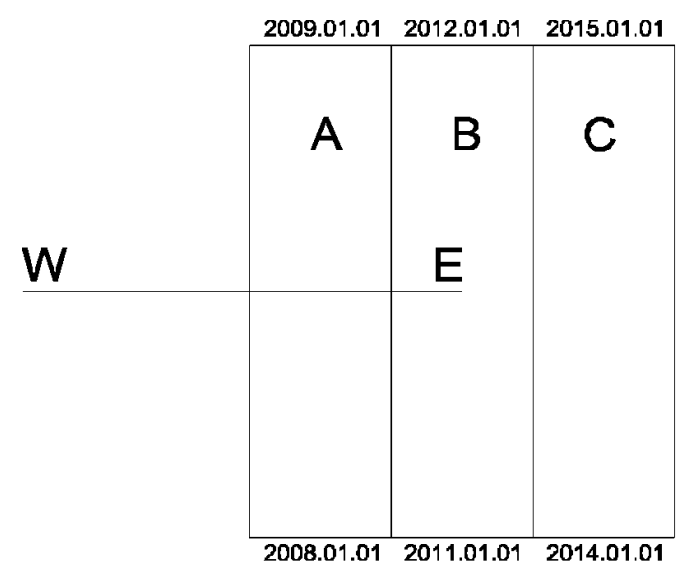

\section{$0 \quad 300 m \quad 600 m$}

Fig. 1 The simulated exploitation used in this study and the location of the line along which deformation indicators and uncertainties were modeled.

Table 1 Average parameters and associated uncertainties for the exploitation of USCB.

\begin{tabular}{lcc}
\hline Parameter & Mean value & Uncertainty \\
\hline$a$ & 0.80 & 0.12 \\
$\tan \beta$ & 1.92 & 0.28 \\
$p=k \cdot H$ & $0.1 \mathrm{H}(=60 \mathrm{~m})$ & $0.05 \mathrm{H}(=30 \mathrm{~m})$ \\
$b_{n}$ & 0.32 & 0.04 \\
\hline
\end{tabular}

The average parameters and associated uncertainties (Table 1) used in this study are based on a previous analysis (Kowalski, 2007) of 29 subsidence basins from different mines across the Upper Silesian Coal Basin (USCB) region, Poland. These basins formed because of the exploitation of beds at different depths, the occurrence of panels with different dimensions (i.e. relative and absolute), and variable geological conditions. At the same time, all of these basins were formed as a result of hard coal deposits longwall mining with caving. The results of this study reveal no correlations between individual parameters and their determined counterparts or exploitation depth. Application of the Shapiro-Wilk test demonstrated that there is no reason to reject the hypothesis that each parameter is normally distributed.

Computations utilizing both LPU and MC methods were performed in this study for subsidence $s$, tilt $T$, and horizontal strain $E$. The calculations assume that influence of all longwalls $(\mathrm{A}, \mathrm{B}, \mathrm{C})$ has been fully revealed.

Ten thousand iterations were performed when using the Monte Carlo method in this study. This number was assumed to be sufficient for the accurate determination of indicator distributions at individual points, as well as for related statistics. There are several reasons that have resulted in the selection of the number of MC trials made. On the one hand, literature studies indicate that using the Monte Carlo method in propagating uncertainties associated with the use of the Knothe model as sufficient to determine basic statistics (average, standard uncertainty), the number of 100 samples was often considered (Niedojadło, 2008; Hejmanowski and Malinowska, 2016). Increasing the number of trials (iterations of the MC) allows both to increase the precision of the statistics generated, as well as reliable modeling of variable distributions in an ever wider range.

The upper limit on the number of MC iterations depends on the time that can be spent on calculations and the required accuracy of the statistics generated. The number of MC trials adopted in the research is the result of these factors. An analysis of the convergence of the values of selected indicator statistics (average, standard deviation) at selected points between samples of 1000 trials indicated that these discrepancies are small enough, i.e. not more than a few percent of the indicator value. On this basis, it was considered that performing more than 10,000 iterations was not justified. The calculation time and the actual accuracy of the deformation model used were also taken into account. The influence of individual parameters on indicator uncertainties and discrepancies between estimates derived from both methods were contrasted. The influence of a particular parameter was analyzed by considering the uncertainties in this variable in isolation to the exclusion of all others.

As similar estimates for standard uncertainties generated using LPU and MC methods do not guarantee a similar basis for probabilistic interpretation, histograms and charts of percentile values and indicator uncertainties were also generated in order to compare both sets of results.

\section{RESULTS}

Average values for parameter indicators along the $\mathrm{W}-\mathrm{E}$ line are presented in Figure 2. These results 

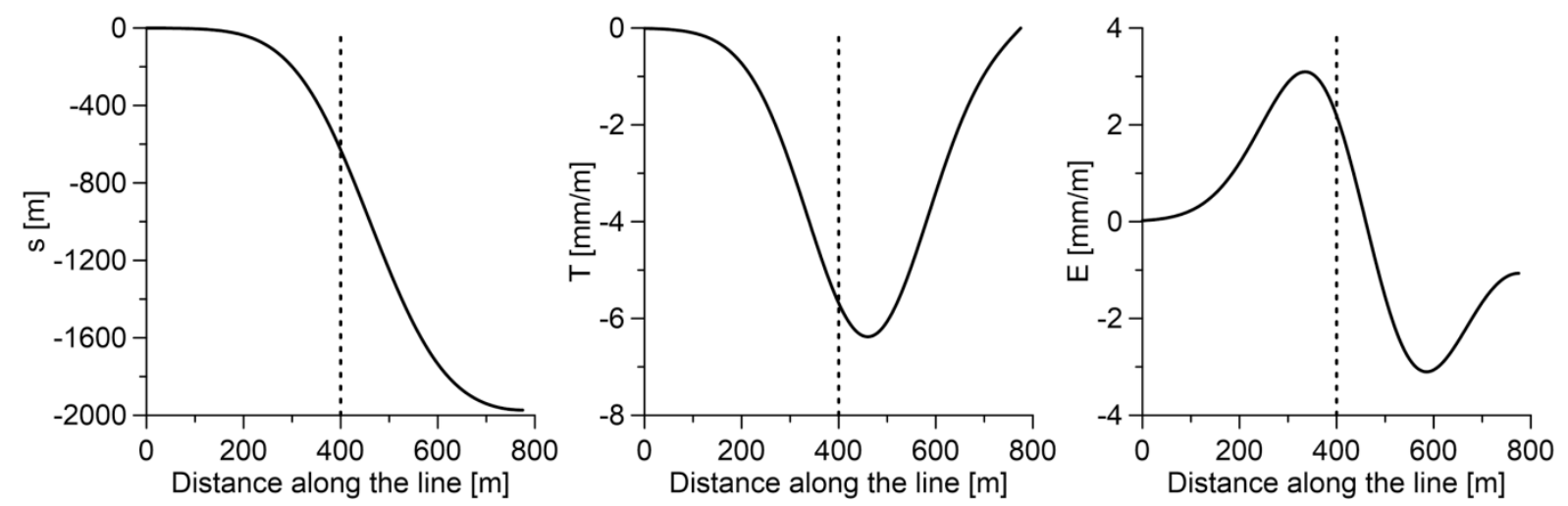

Fig. 2 Deformation indicator values for mean parameters along the W-E line.
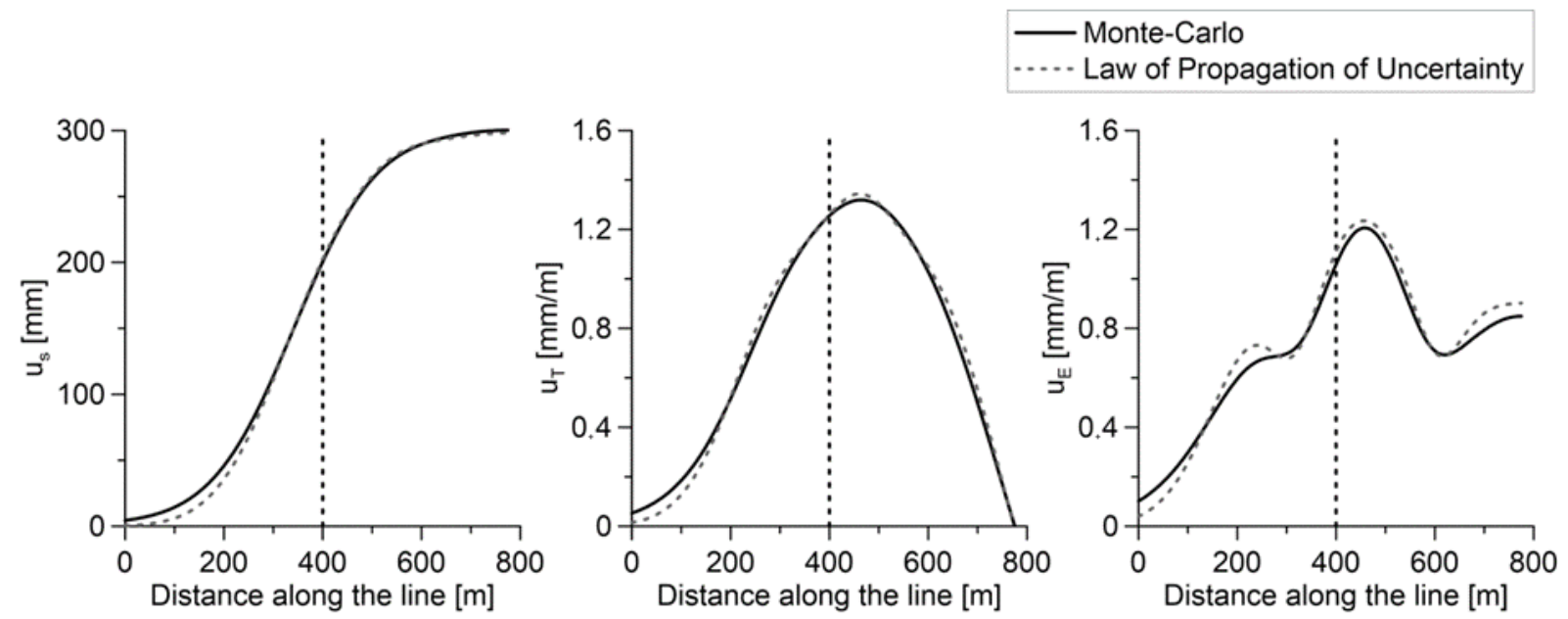

Fig. 3 Estimated uncertainties in deformation indicators.

show that subsidence values along the observation line vary between zero at one end to approximately $2000 \mathrm{~mm}$ at the other, while tilt values reach a maximum of approximately $-6.5 \mathrm{~mm} / \mathrm{m}$ at a distance of $60 \mathrm{~m}$ from the exploitation edge (marked with a vertical dashed line on the graphs in Fig. 2). That shift of this maximum tilt value from above the field edge towards the center of the exploitation area is related to the influence of the operating rim; the extreme horizontal strains for mean parameters were $+3.1 \mathrm{~mm} / \mathrm{m}$ and $-3.1 \mathrm{~mm} / \mathrm{m}$, respectively.

Calculated deformation indicator uncertainties along the same observation line are presented in Figure 3.

Results show that both relative and absolute discrepancy values for estimated subsidence uncertainties based on both methods decrease with increases in uncertainty and indicator values for average model parameters. In contrast, discrepancies for tilt and horizontal strain uncertainty estimates are harder to interpret. Maximum uncertainty discrepancies determined using both methods are compared in Table 2.
Table 2 Maximum discrepancies between uncertainties estimated using the LPU and MC methods.

\begin{tabular}{lc}
\hline \multicolumn{1}{c}{ Indicator } & $\begin{array}{c}\text { Maximum absolute } \\
\text { difference }\end{array}$ \\
\hline$s[\mathrm{~mm}]$ & 10.5 \\
$T[\mathrm{~mm} / \mathrm{m}]$ & 0.060 \\
$E[\mathrm{~mm} / \mathrm{m}]$ & 0.079 \\
\hline
\end{tabular}

The values of these discrepancies are not high and can therefore be considered negligible in practice for this type of mining.

The results of this study show that, in terms of subsidence, accuracy in determining exploitation coefficient $a$ exerts the most significant influence on uncertainty (Fig. 4), while all parameter values (i.e., $a, \tan \beta, p)$ are similarly important in the case of tilt (Fig. 4 Fig.6). Indeed, the influence of exploitation coefficient $a$ and $\tan \beta$ are most important at extreme values of tilt, while accuracy in determining 

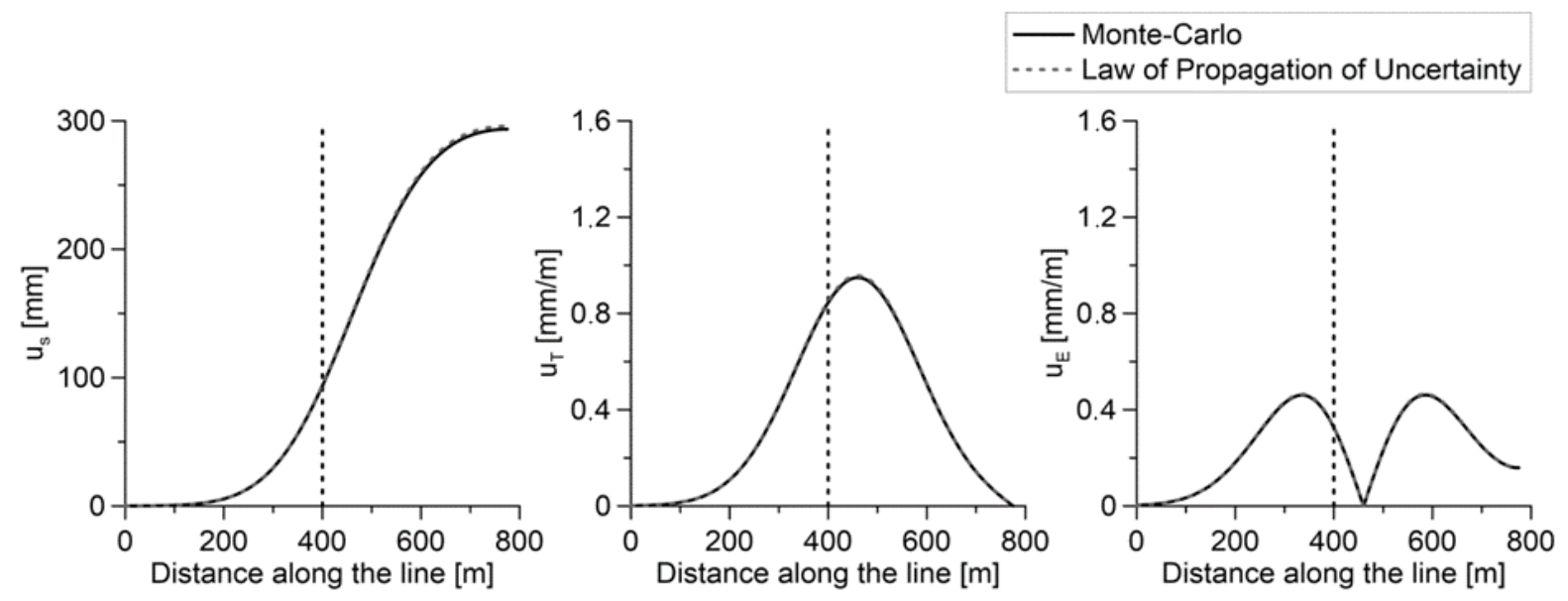

Fig. 4 The influence of $a$ parameter uncertainty on deformation indicator uncertainties.
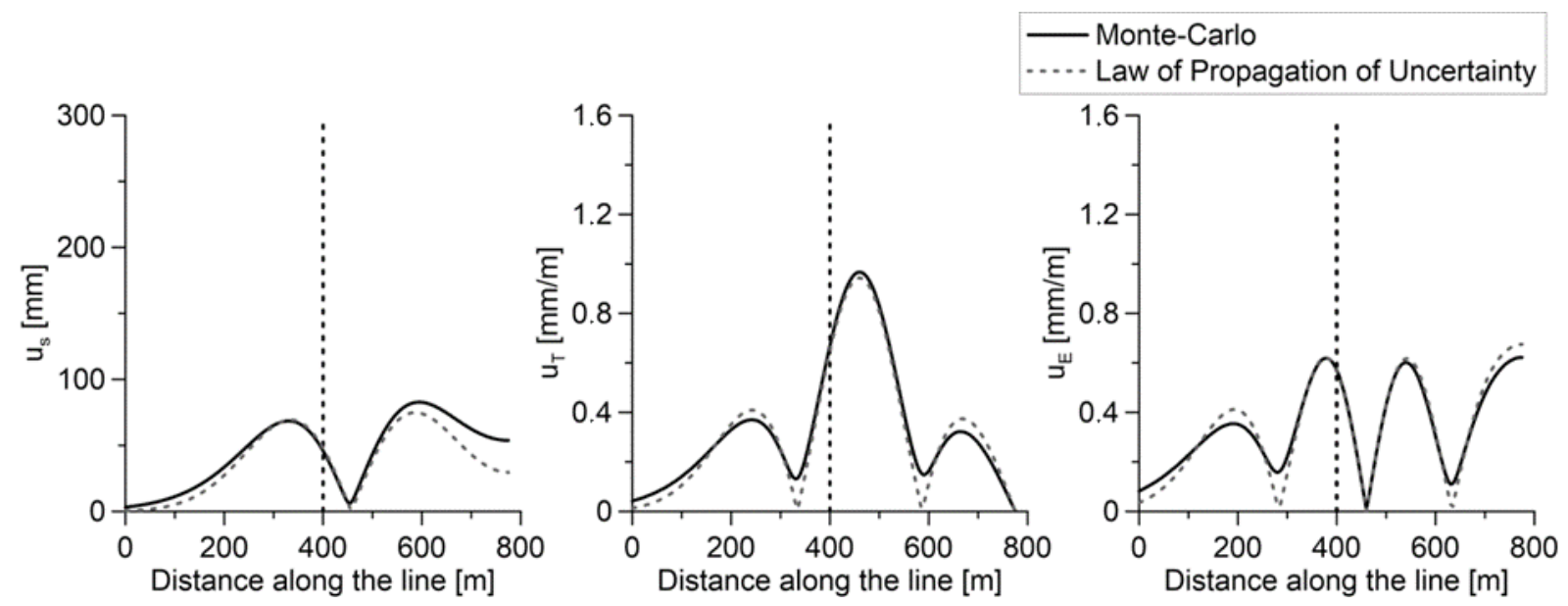

Fig. 5 The influence of $\tan \beta$ parameter uncertainty on deformation indicator uncertainties.

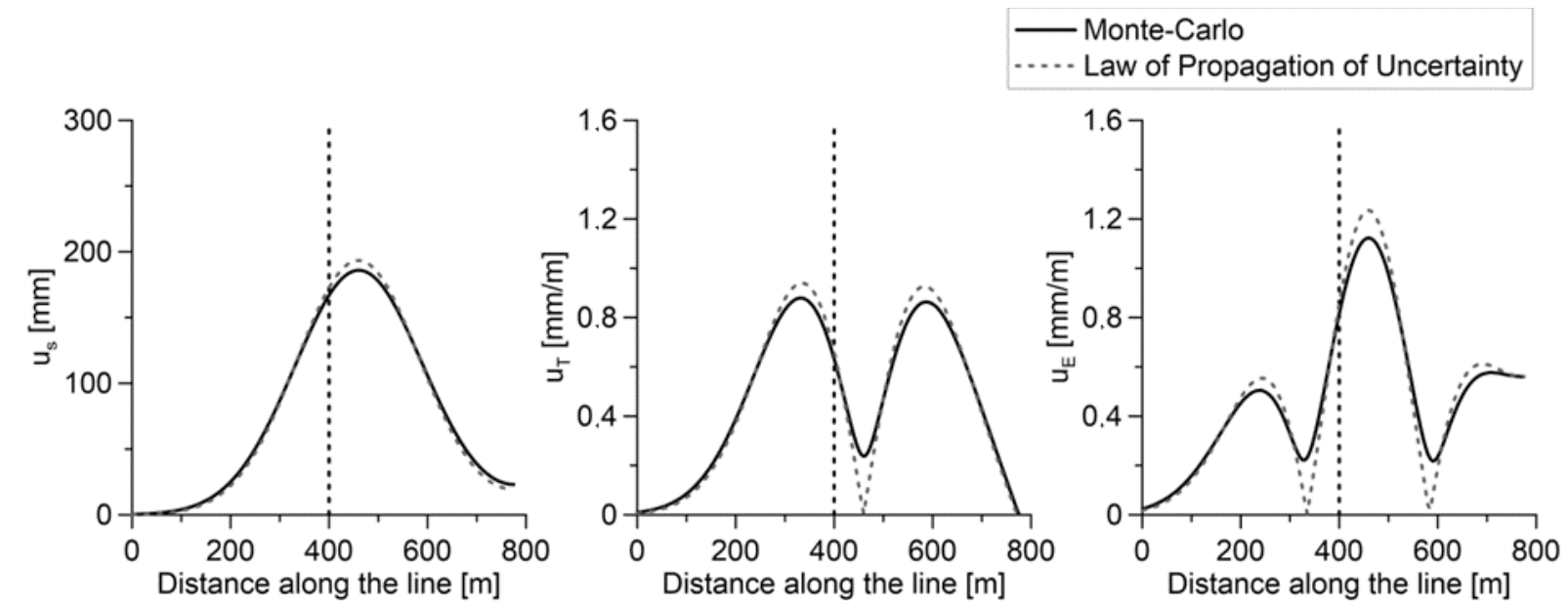

Fig. 6 The influence of $p$ parameter uncertainty on deformation indicator uncertainties. 
operating rim $p$ is crucial at certain distances from the effective mining field edge. The influence of operating rim accuracy determination is paramount in terms of horizontal strain uncertainties.

Comparing the results from both LPU and MC methods necessitates the determination of discrepancies between indicator uncertainties, as well as a comparison of their probabilistic interpretations. In cases where the LPU method is used, a normal distribution of indicator values at a given point and under certain exploitation conditions is frequently assumed as default. A series of exemplary histograms of deformation indicator values at point distances of $200 \mathrm{~m}$ (Fig. 8) and $400 \mathrm{~m}$ (Fig. 9) from the start of the $\mathrm{W}-\mathrm{E}$ line were generated in this study to verify the correctness of this assumption using $\mathrm{MC}$ simulation results. Against the background of these histograms, the probability density function estimated by kernel density method (i.e. a normal kernel function), as well as the fit of the normal distribution function are presented. Discrepancies between these functions illustrate deviation from the assumption of normality; skewed indicator distributions are particularly noticeable at a distance of $200 \mathrm{~m}$ from the beginning of the W-E line (Fig. 8).

The discrepancies revealed by these results mean that both the normality assumption and related probabilistic interpretations deviate significantly from reality, at least in cases where indicator values at the western end of the W-E line are low. In order to verify the practical importance of the non-normality of these distributions, a series of comparative charts for percentiles and means $+/$ - standard uncertainty for the deformation indicators analyzed in this study were generated (Fig. 10). Percentiles were determined using the MC method while uncertainties were computed using the LPU method. Mean values +/- standard uncertainty are approximately equal to 16 and 84 percentiles, respectively; thus, the probability of locating an actual indicator value within this range

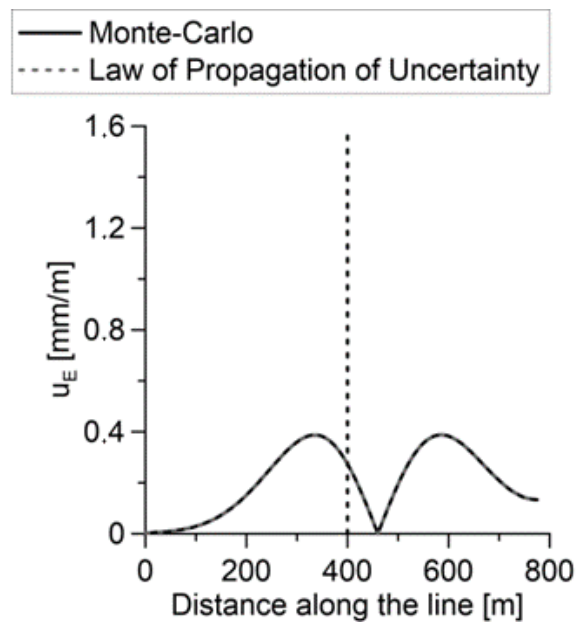

Fig. 7 The influence of parameter uncertainty $b_{r}$ on horizontal strain uncertainties.
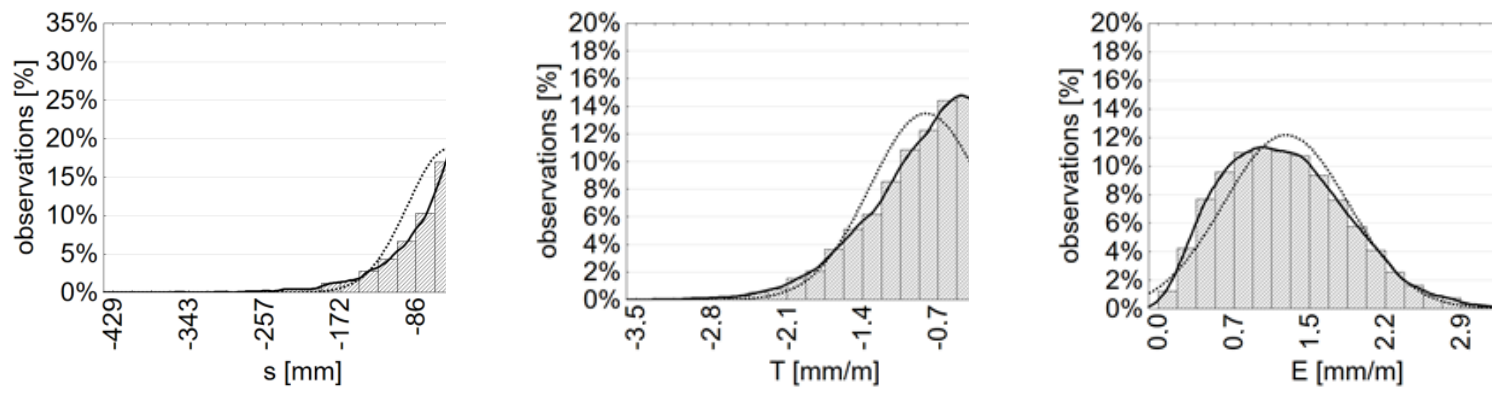

Fig. 8 Histograms and distributions of deformation indicators at a point $200 \mathrm{~m}$ from the beginning of the W-E line (i.e. outside the rim).
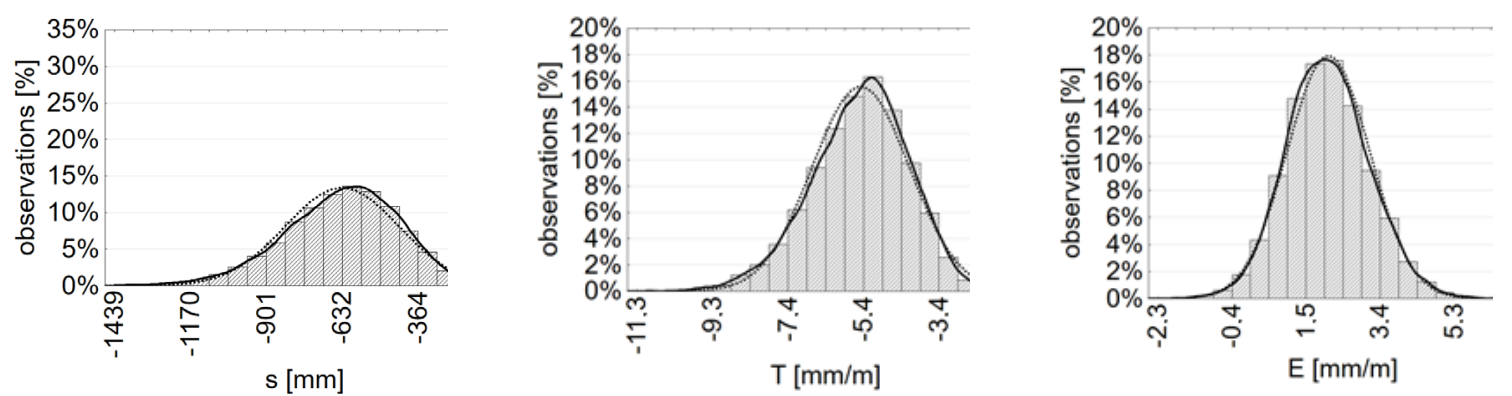

Fig. 9 Histograms and distributions of deformation indicators at a point $400 \mathrm{~m}$ from the beginning of the W-E line (i.e. above the rim). 

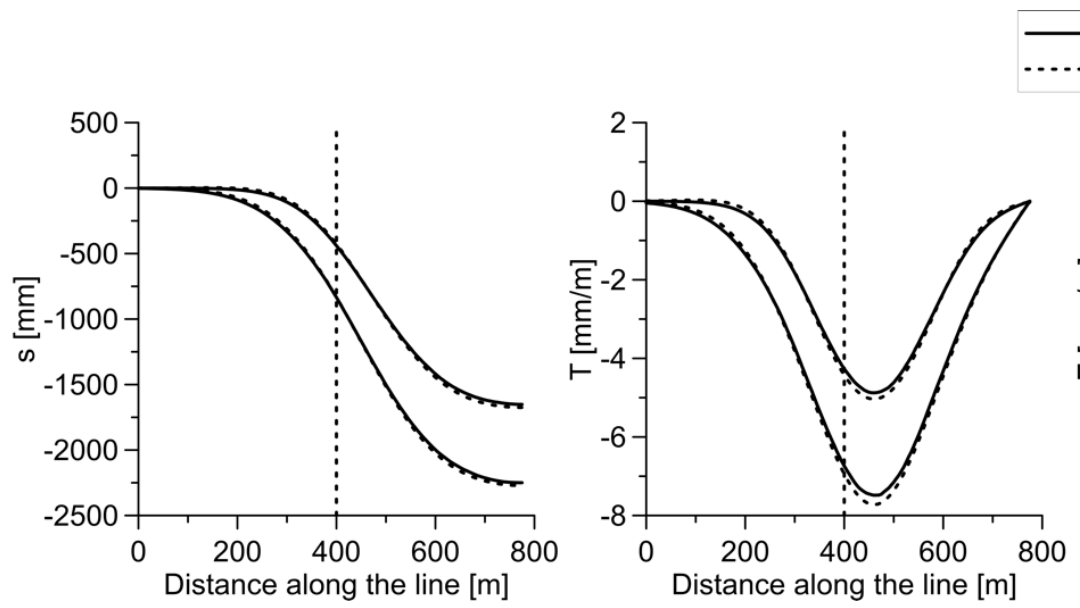

M-C, percentiles $16 \%, 84 \%$

LPU, mean+/-standard uncertainty

Fig. 10 Percentile values determined using the MC method, as well as means +/- standard uncertainty determined using the LPU.
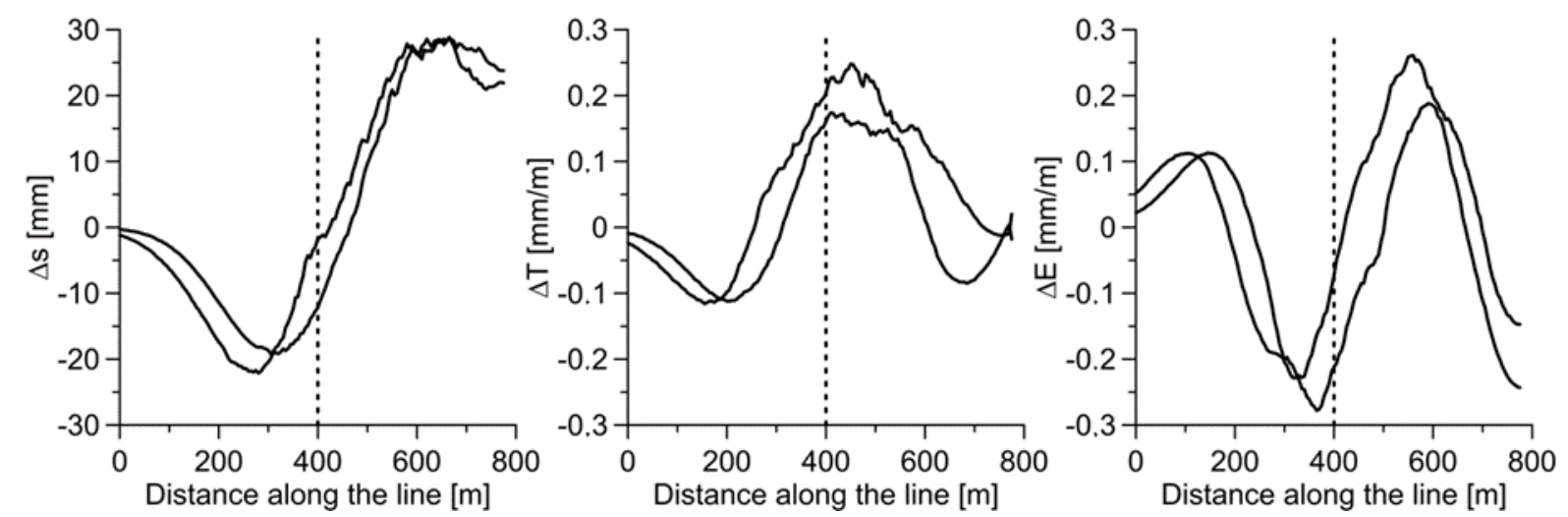

Fig. 11 Comparison of discrepancies between 16 and 84 percentile values estimated using the MC method and means $+/$ - standard uncertainty estimated using the LPU.

(68\%) corresponds to the approximate probability of locating an actual value within the range of the mean indicator +/- standard uncertainty, given the assumption of a normal indicator distribution at a given point.

Results show that discrepancies between values tend to be low (Fig. 11). A comparison of maximum values is presented in Table 3. It is noteworthy that percentile values generated in this study are 'symmetric' with respect to the median rather than to average indicator values. In the case of asymmetrical distributions the average and median do not overlap, the average is shifted towards the tail of the distribution.

\section{DISCUSSION}

Parameter uncertainty is just one factor that can lead to non-compliance between predicted and actual deformation factors; another example is random scatter of observed indicator values.

Previous studies that have been carried out in Poland on the random scatter of deformation
Table 3 Maximum discrepancies between percentile values estimated using the MC method and uncertainties estimated using the LPU.

\begin{tabular}{ccc}
\hline Indicator & $\begin{array}{c}\text { Maximum } \\
\text { discrepancy }\end{array}$ & $\begin{array}{c}\text { Indicator value for mean } \\
\text { parameters at a given point }\end{array}$ \\
\hline$S[\mathrm{~mm}]$ & 29 & -1895 \\
$T[\mathrm{~mm} / \mathrm{m}]$ & 0.25 & -6.4 \\
$E[\mathrm{~mm} / \mathrm{m}]$ & 0.27 & +2.9 \\
\hline
\end{tabular}

indicators (Kowalski, 2007; Stoch, 2005) have utilized the variability coefficient, $M_{D}$ by Equation 7 :

$M_{D}=\frac{\sigma_{D}}{\left|D_{\max }^{\text {approx }}\right|} \cdot 100 \%$ 
Table 4 Values for the variability coefficient $M_{D}$.

\begin{tabular}{lcc}
\hline Indicator $D$ & $M_{D}$ estimated by Kowalski [\%] & $M_{D}$ estimated by Stoch [\%] \\
\hline$S$ & \pm 3 & \pm 1 \\
$T$ & \pm 9 & \pm 7 \\
$E$ & \pm 25 & \pm 23 \\
\hline
\end{tabular}

Table 5 Estimated values for deformation indicator uncertainties that result from random scatter.

\begin{tabular}{lcc}
\hline Indicator & Maximal value & Estimated scatter-related uncertainty \\
\hline$s[\mathrm{~mm}]$ & -1973 & 39 \\
$T[\mathrm{~mm} / \mathrm{m}]$ & -6.4 & 0.5 \\
$E[\mathrm{~mm} / \mathrm{m}]$ & -3.1 & 0.7 \\
\hline
\end{tabular}

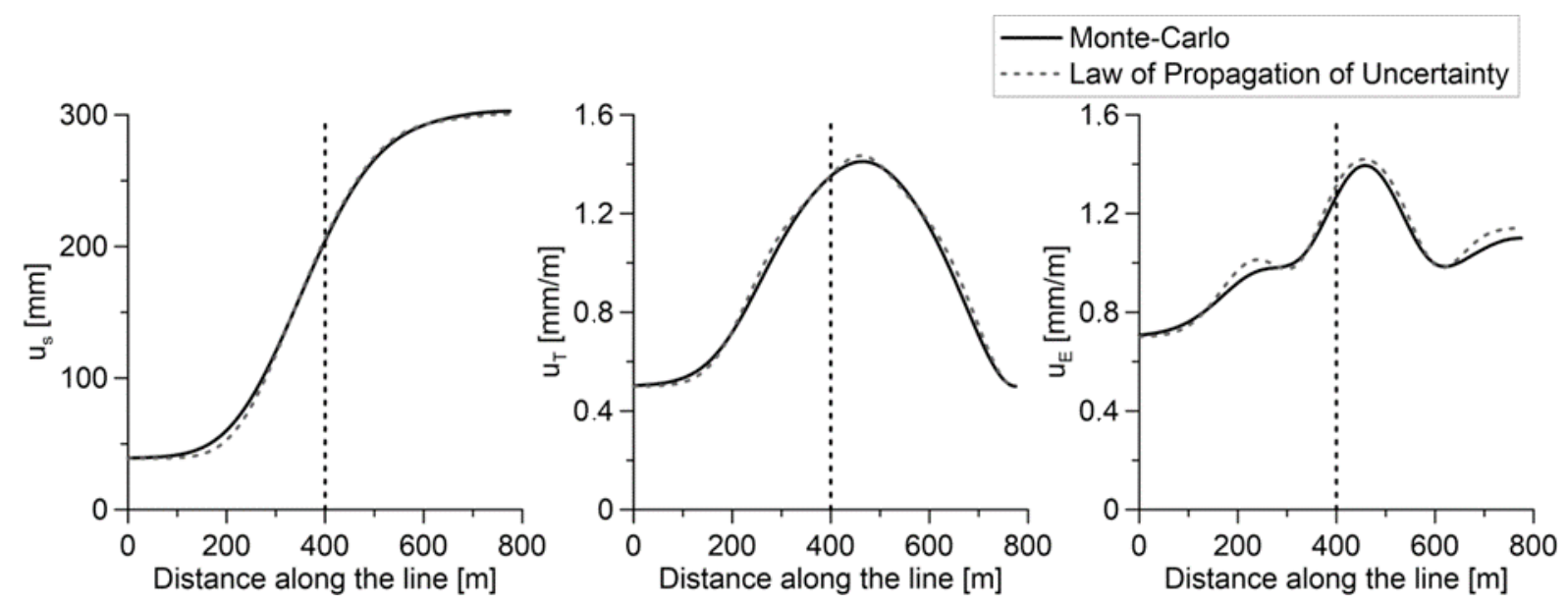

Fig. 12 Estimated deformation indicator uncertainties when random scatter is taken into account.

In this expression, $\sigma_{D}$ denotes the standard deviation of a deformation indicator from conditional average values modeled as seamless (smooth) indicator flow, while $D_{\max }^{a p p r o x}$ is the maximum modeled value of a given indicator.

The coefficient $M_{D}$ therefore denotes the standard deviation value of deformation indicator $D$ as a percentage of its maximum modeled value. Variability coefficient values for selected deformation indicators based on studies performed on the basis of observations from the USCB region by Stoch (Stoch, 2005) and Kowalski (Kowalski, 2007) are presented in Table 4.

On this basis the random scatter of deformation indices was estimated for simulated exploitation (Table 5). The uncertainties of the predicted deformation indices resulting only from their random scatter are greater than the previously described discrepancies between the results of both methods. This applies to both the comparison of the uncertainties themselves (Table 2, Figure 3) and the comparison of the conditional distributions of the indicators taking into account non-normality (Table 3, Figures 10, 11).

Indicator uncertainty values estimated using both methods and taking random scatter into account are presented in Figure 12.

These results reveal a relative decrease in the importance of discrepancies between the two propagation methods when this additional factor is incorporated.

While reducing the uncertainty of model parameters, such as when the forecast is based on ongoing surveys, the influence of parameter uncertainty on the uncertainty of the indicators will also be reduced. In such a case, the significance of the indicators random scatter will increase. At the same time, discrepancies between the uncertainties of indicators determined by different propagation methods will also decrease. 
Therefore, the question should be asked about the reasonableness of using Monte Carlo simulation in the propagation of uncertainty. This method generates a much larger computational effort than the application of the law of propagation of uncertainty even if the number of iterations is much smaller than in the described studies. The use of MC simulation instead the law of propagation of uncertainty seems to make sense only for the distribution of parameters significantly different from the normal one. In this case, this method will allow for estimating distributions of deformation indices and probabilistic interpretation, which will not be ensured by the law of propagating uncertainty.

\section{CONCLUSIONS}

As a result of the research, the following synthetic conclusions were made:

1. Discrepancies between deformation indicator uncertainties estimated using LPU and MC methods are negligible in practical situations if normal probability distributions for model parameters are assumed;

2. Due to computational load application of the LPU is recommended when deformation model parameter uncertainties conform to a normal distribution, and;

3. With normal model parameter distributions, the assumption of normality of indicator distributions is sufficiently accurate for the purpose of forecasts.

\section{ACKNOWLEDGMENTS}

This work was supported by statutory research funds from the Faculty of Mining Surveying and Environmental Engineering AGH-UST no. 16.16.150.545 (Department of Mining Areas Protection, Geoinformatics and Mining Surveying).

\section{REFERENCES}

Aien, M., Hajebrahimi, A. and Fotuhi-Firuzabad, M.: 2016, A comprehensive review on uncertainty modeling techniques in power system studies. Renewable and Sustainable Energy Reviews, 57, 1077-1089.

DOI: 10.1016/j.rser.2015.12.070

Aien, M., Rashidinejad, M. and Fotuhi-Firuzabad, M.: 2014 On possibilistic uncertainty assessment of power flow problem, A review and a new approach. Renew. Sust. Energ. Rev., 37, 883-895.

DOI: $10.1016 /$ j.rser.2014.05.063

Gruszczyński, W., Niedojadło, Z. and Mrocheń, D.: 2018, Influence of model parameter uncertainties on forecasted subsidences. Acta Geodyn. Geomater., 15, 3, 211-228. DOI: 10.13168/AGG.2018.0016

Gruszczyński, W., Niedojadło, Z. and Mrocheń, D.: 2019, Uncertainty in determining the parameters of the surface deformation model. Acta Geodyn. Geomater., 16, 2, 211-218. DOI: 10.13168/AGG.2019.0017
Hejmanowski, R. and Malinowska, A.A.: 2016, Significance of the uncertainty level for the modeling of ground deformation ranges. Int. J. Rock Mech. Min., 83, 140 148. DOI: $10.1016 /$ j.ijrmms.2015.12.019

Knothe, S.: 1957, Observations of surface movements under influence of mining and their theoretical interpretation. In: Proceeding European Congress on Ground Movement, Leeds, 210-218.

Kowalski, A.: 2005, Errors of surface deformations factors due to errors of parameters of theories. In: Kwiatek, J. (Eds): Problems of mining exploitation under urban areas. Central Mining Institute, Katowice, 270-277, (in Polish).

Kowalski, A.: 2007, Transient mining surface deformations in the prediction accuracy aspect. Central Mining Institute, Pap No. 871, Katowice, (in Polish).

Kwinta, A.: 2012, Procedure of determination of Knothe theories parameters. In: Kowalski, A. (Eds): Objects protection on mining areas. Central Mining Institute, Katowice, 171-179, (in Polish).

Metropolis, N. and Ulam, S.: 1949, The Monte Carlo method. J. Am. Stat. Assoc., 44, 247, 335-341.

Naworyta, W., Menz, J. and Sroka, A.: 2005, Assessment of the accuracy of ground movement elements prediction using simulation method. Proceedings of 6th International Mining Forum, 123-126.

Niedojadło, Z.: 2008, The problems of exploitation of the copper deposit in shaft protection pillars in the conditions of LGOM. Dissertations and Monographs No. 177, Cracow, 176 pp., (in Polish).

Niedojadło, Z. and Gruszczyński, W.: 2015, The impact of the estimation of the parameters values on the accuracy of predicting the impacts of mining exploitation. Arch. Min. Sci., 60, 1, 173-193. DOI: $10.1515 / \mathrm{amsc}-2015-0012$

Soroudi, A. and Amraee, T.: 2013, Decision making under uncertainty in energy systems, state of the art. Renew. Sust. Energ. Rev., 28, 376-384. DOI: 10.1016/j.rser.2013.08.039

Stoch, T.: 2005, Impact of mining and geological conditions of mining extraction on randomness of displacement and surface deformation. Ph.D. thesis, Cracow, AGHUST, (unpublished, in Polish).

Taylor, J.R.: 1997, An introduction to error analysis: the study of uncertainties in physical measurements. 2nd edition. University Science Books, Sausalito, California. 